\title{
Existence of continuous right inverses to linear mappings in finite-dimensional geometry
}

\author{
Christer Oscar Kiselman • Erik Melin
}

Received: 27 May 2020 / Accepted: 29 July 2020 / Published online: 19 October 2020

(C) The Author(s) 2020

\begin{abstract}
A linear mapping of a compact convex subset of a finite-dimensional vector space always possesses a right inverse, but may lack a continuous right inverse, even if the set is smoothly bounded. Examples showing this are given, as well as conditions guaranteeing the existence of a continuous right inverse.
\end{abstract}

Keywords Linear mapping · Continuous right inverse $\cdot$ Projection · Finitedimensional vector space

\section{Existenz von stetigen Rechtsinversen zu linearen Abbildungen in der endlich-dimensionaler Geometrie}

Zusammenfassung Eine lineare Abbildung einer kompakten konvexen Menge in einem endlich-dimensionalen Vektorraum hat immer eine Rechtsinverse, aber nicht notwendigerweise eine stetige Rechtsinverse, auch dann nicht wenn der Rand der Menge glatt ist. Wir geben Beispiele dafür, sowie hinreichende Voraussetzungen für die Existenz einer stetigen Rechtsinversen.

\section{Introduction}

A mapping has a right inverse if and only if it is surjective-at least if we allow the use of the axiom of choice. However, it is of interest to have not only a right inverse

C. O. Kiselman $(\bowtie)$

Department of Information Technology, Uppsala University, P.O. Box 337, 75105 Uppsala, Sweden

E-Mail: kiselman@it.uu.se; christer@kiselman.eu

E. Melin

Eksoppsvägen 16, 75646 Uppsala, Sweden

E-Mail: erik@melin.nu 
to a surjective mapping, but also a right inverse with some additional properties, like continuity. In this note we shall study the question of continuity for linear mappings in finite-dimensional vector spaces.

An indication of the interest for this question is the book with its many references by Repovš \& Semenov [4] on the selection of a continuous mapping inside a given multivalued mapping.

Let $F: \mathbf{R}^{n} \rightarrow \mathbf{R}^{m}$ be a linear mapping. Let $A$ be a subset of $\mathbf{R}^{n}$ and $B=$ $\{F(x) ; x \in A\}$ its image in $\mathbf{R}^{m}$ under $F$. We denote by $f: A \rightarrow B$ the restriction of $F$ to $A$ and ask whether $f$ has a right inverse which is continuous for the usual vector space topologies on $\mathbf{R}^{n}$ and $\mathbf{R}^{m}$, thus whether there exists a continuous mapping $v: B \rightarrow A$ such that $f \circ v=\mathbf{i d}_{B}$. The answer is no in general as we shall see in Sect. 2, even if we assume that $A$ is compact and convex with smooth boundary. Then we prove some results in the positive direction.

If $A$ is convex, then so is its image $B$. If $A$ is compact, then so is $B$ as the image of a compact set under a continuous mapping. If $A$ is open and if we choose as the codomain the smallest vector space containing the image of $F$, then also $B$ is open.

In studying this problem we may assume that $F$ is a projection $\left(x_{1}, \ldots, x_{n}\right) \mapsto$ $\left(x_{1}, \ldots, x_{m}\right)$ for some numbers $0 \leqslant m \leqslant n$. If $m=n$, then $f: A \rightarrow B=A$ is the identity and equal to its own inverse. If $A$ is compact and convex and $m \leqslant 1$, then the existence of such an inverse is also clear. The problem is therefore of interest only if $1<m<n$; there is a counterexample with the minimal dimensions $m=2$ and $n=3$.

In Sect. 2 we present some examples of convex sets where a linear mapping does not have a continuous right inverse. Sect. 3 prepares for the following Sect. 4, where criteria for the existence of a continuous right inverse are established. These criteria are then applied to open and closed sets in the next two sections. The special case of polyhedra is considered in Sect. 7 and an easy sufficient condition for the existence of continuous right inverses is mentioned in Sect. 8. Finally, in Sect. 9, it is noticed that, after all, projections that do not have a continuous right inverse are exceptional in a precise sense.

\subsection{Notation}

We shall use the customary symbols $\mathbf{N}=\{0,1, \ldots\}$ for the semiring of natural numbers and $\mathbf{R}$ for the field of real numbers. By

$$
\mathbf{R}_{!}=\mathbf{R} \cup\{-\infty,+\infty\}=[-\infty,+\infty]
$$

we denote the set of extended real numbers, adding two infinities.

Given any mapping $f: X \rightarrow Y$ we define the inverse image of $B$ and the direct image of $A$ under $f$ by

$$
\begin{aligned}
f^{*}(B) & =\{x \in X ; f(x) \in B\}, & & B \subset Y, \text { and } \\
f_{*}(A) & =\{f(x) \in Y ; x \in A\}, & & A \subset X .
\end{aligned}
$$


We shall write $A \backslash B=\{x \in A ; x \notin B\}$ for the set-theoretical difference of two sets, and $A^{\circ}, \bar{A}$ and $\partial A=\bar{A} \backslash A^{\circ}$ for, respectively, the interior, the closure, and the boundary of a set $A$ in a topological space.

\section{Examples}

We shall present three examples of projections that do not admit a continuous right inverse. The first is simple, and the third, less simple, has a smooth boundary.

Example 1 Let $A$ be the convex hull of a helix in $\mathbf{R}^{3}$,

$$
\left\{(\cos t, \sin t, t) \in \mathbf{R}^{3} ; t \in[0,2 \pi]\right\},
$$

and let $f$ be the restriction to $A$ of the mapping $F:(x, y, z) \mapsto(x, y)$.

It is clear that $A$ contains the segment $[(1,0,0),(1,0,2 \pi)]$ as well as the triangle $T(t)$ with vertices at $(1,0,0),(1,0,2 \pi),(\cos t, \sin t, t)$ for every $t$ with $0<t<2 \pi$. Actually $A$ is equal to the union of these sets. The convex hull of the union $T\left(t_{0}\right) \cup$ $T\left(t_{1}\right)$ of two such triangles is a tetrahedron with vertices in $(1,0,0),(1,0,2 \pi)$, $p^{(0)}=\left(\cos t_{0}, \sin t_{0}, t_{0}\right)$ and $p^{(1)}=\left(\cos t_{1}, \sin t_{1}, t_{1}\right)$. We have $\left\|p^{(j)}-\left(0,0, t_{j}\right)\right\|_{2}=$ $1, j=0,1$, which implies that any point $p^{(s)}=(1-s) p^{(0)}+s p^{(1)}, 0 \leqslant s \leqslant 1$, on the segment $\left[p^{(0)}, p^{(1)}\right]$ satisfies, writing $t_{s}=(1-s) t_{0}+s t_{1}$,

$$
\begin{aligned}
\left\|p^{(s)}-\left(0,0, t_{s}\right)\right\|_{2} & =\left\|(1-s)\left(p^{(0)}-\left(0,0, t_{0}\right)\right)+s\left(p^{(1)}-\left(0,0, t_{1}\right)\right)\right\|_{2} \\
& \leqslant(1-s)\left\|p^{(0)}-\left(0,0, t_{0}\right)\right\|_{2}+s\left\|p^{(1)}-\left(0,0, t_{1}\right)\right\|_{2}=1 .
\end{aligned}
$$

Thus the convex hull of $T\left(t_{0}\right) \cup T\left(t_{1}\right)$ is contained in the union of all the triangles $T(t)$ as claimed, proving that this union together with the segment $[(1,0,0),(1,0,2 \pi)]$ is a convex set. This also proves that any right inverse $v$ to $f$ must satisfy $v(\cos t, \sin t)=(\cos t, \sin t, t)$ when $t \in] 0,2 \pi$ [; there cannot be any other choice of the value of $v$. It also follows that $v$ cannot be continuous at $(1,0)$.

We note that $f$ is injective near every point $(\cos t, \sin t, t)$ with $0<t<2 \pi$ but not at the points $(1,0, t), 0 \leqslant t \leqslant 2 \pi$. The inverse image $f^{*}(\{(1,0)\})$ of $(1,0)$ is the whole segment $[(1,0,0),(1,0,2 \pi)]$.

Example 2 In the above example $A$ does not have a smooth boundary. We may improve the smoothness by considering the dilation $A_{r}=A+B_{\leqslant}(0, r)$, where $B_{\leqslant}(0, r)$ denotes the closed ball af radius $r$ centered at the origin, and where $0<$ $r<1$. Then $A_{r}$ has a $C^{1}$ boundary. As in the previous example, the projection of $A_{r}$ into $\mathbf{R}^{2}$ cannot have a continuous right inverse, now at the point $(1+r, 0)$, which belongs to the boundary of $f_{*}\left(A_{r}\right)$.

Is it possible to have even a $C^{\infty}$ boundary? Indeed this is the case as we shall see now. 
Example 3 Let $\varphi(x)=x \mathrm{e}^{-1 / x}$ for $x$ positive, $\varphi(0)=0$ and $\varphi(-x)=\varphi(x)$. This function is of class $C^{\infty}$ and convex on all of $\mathbf{R}$. We define

$$
g(x, z)=a x^{2}+\varphi(x)(z-b \operatorname{sgn}(x))^{2}, \quad(x, z) \in \mathbf{R}^{2} .
$$

Here sgn is the sign function, taking the value -1 for $x<0,0$ for $x=0$, and 1 for $x>0, a$ is a sufficiently large positive constant, and $b$ is a real number, $0 \leqslant b \leqslant 1$. Then the infimum when $z$ varies is attained at $z=b$ when $x>0$, at $z=-b$ when $x<0$ and at every real $z$ when $x=0$. We calculate the Hessian determinant of $g$ and find that it is, for $x>0$,

$$
\begin{aligned}
h=g_{x x} g_{z z}-\left(g_{x z}\right)^{2} & =\left(2 a+(z-b)^{2} \varphi^{\prime \prime}\right)(2 \varphi)-\left(2(z-b) \varphi^{\prime}\right)^{2} \\
& =2 \varphi\left(2 a+(z-b)^{2}\left(\varphi^{\prime \prime}-2\left(\varphi^{\prime}\right)^{2} / \varphi\right)\right) .
\end{aligned}
$$

Here

$$
\varphi^{\prime \prime}(x)-2 \varphi^{\prime}(x)^{2} / \varphi(x)=-\mathrm{e}^{-1 / x}\left(x^{-3}+4 x^{-2}+2 x^{-1}\right) \geqslant-M
$$

for some positive constant $M$. When $z \in[-2,2]$, we have $(z-b)^{2} \leqslant 9$, so to get $h$ nonnegative for all $z \in[-2,2]$ it is enough to choose $a$ so large that $2 a-9 M \geqslant 0$. Since also $g_{x x}$ is nonnegative, $g$ is convex in $\mathbf{R} \times[-2,2]$. We construct a bounded set in $\mathbf{R}^{3}$ as follows.

We define

$$
H(x, y, z)=g(x, z)+\psi(z)+a(y-r)^{2}-a r^{2}, \quad(x, y, z) \in \mathbf{R}^{3},
$$

where $r$ is a positive number to be chosen later, and $\psi$ is even and defined as $\psi(z)=\varphi(z-1)$ for $z \geqslant 1$, zero on $[-1,1]$. Hence $\psi$ is positive outside this interval.

We now define

$$
\begin{aligned}
& A=\left\{(x, y, z) \in \mathbf{R}^{3} ; H(x, y, z) \leqslant 0\right\}, \\
& B=\left\{(x, y) ; \inf _{z} H(x, y, z) \leqslant 0\right\}=\left\{(x, y) ; x^{2}+(y-r)^{2} \leqslant r^{2}\right\},
\end{aligned}
$$

and let $f$ be the projection $A \rightarrow B$ defined by $f(x, y, z)=(x, y)$. Clearly $f$ does not admit a continuous right inverse if $b \neq 0$, since any right inverse $v: B \rightarrow A$ must satisfy $v(x, y)=(x, y, b \operatorname{sgn}(x))$ when $x \neq 0$ and $y$ is such that $x^{2}+(y-r)^{2}=r^{2}$.

For a suitable choice of $r, H$ is convex where it is nonpositive. Indeed, $H \leqslant 0$ implies that $\psi(z) \leqslant a r^{2}$, so if we choose $r$ so small that $\psi(2) \geqslant a r^{2},(x, y, z) \in A$ implies $z \in[-2,2]$ and we see that $H$ is convex in $A$.

Furthermore, the set $A$ has a smooth boundary. For when $x \geqslant 0$, the gradient of $H$ vanishes only when $x=0, y=r$, and $z=1$, and then $H$ takes the value $-a r^{2} \neq 0$. 


\section{Upper and lower inverses}

If $f: X \rightarrow Y$ and $g: Y \rightarrow Z$ are two mappings with right inverses $u$ and $v$, then $u \circ v$ is a right inverse to $g \circ f$. Indeed,

$$
(g \circ f) \circ(u \circ v)=g \circ(f \circ u) \circ v=g \circ \mathbf{i d}_{Y} \circ v=\mathbf{i d}_{Z} .
$$

When $F: \mathbf{R}^{n} \rightarrow \mathbf{R}^{m}$ is the projection which forgets the last $n-m$ coordinates, we can use this observation to reduce the projection to a composition of $n-m$ projections each forgetting only one coordinate, thus to the case $m=n-1$ : we project from $\mathbf{R}^{n}$ to $\mathbf{R}^{n-1}$, then from $\mathbf{R}^{n-1}$ to $\mathbf{R}^{n-2}$ and so on, until we reach $\mathbf{R}^{m}$.

Definition 1 If $m=n-1$ and $A$ is any subset of $\mathbf{R}^{n}$, we define two functions $u_{A}, w_{A}: \mathbf{R}^{m} \rightarrow \mathbf{R}$ ! by taking the infimum and supremum of the last coordinate, respectively, i.e.,

$$
\begin{aligned}
& u_{A}(x)=\inf _{t \in \mathbf{R}}(t \in \mathbf{R} ;(x, t) \in A) \text { and } \\
& w_{A}(x)=\sup _{t \in \mathbf{R}}(t ;(x, t) \in A), \quad x \in \mathbf{R}^{m} .
\end{aligned}
$$

If the inverse image $f^{*}(\{x\})$ of every point in $\mathbf{R}^{m}$ is connected, we have

$$
\begin{aligned}
& \left\{(x, t) \in \mathbf{R}^{m} \times \mathbf{R} ; u_{A}(x)<t<w_{A}(x)\right\} \subset A \\
& \subset\left\{(x, t) \in \mathbf{R}^{m} \times \mathbf{R} ; u_{A}(x) \leqslant t \leqslant w_{A}(x)\right\} .
\end{aligned}
$$

If $A$ is compact, the restrictions of $u_{A}$ and $w_{A}$ to $B=f_{*}(A)$ are actually right inverses. We shall then call them the lower and upper right inverses.

Let as before $f: A \rightarrow B$ be the restriction to $A$ of the projection $F: \mathbf{R}^{n} \rightarrow \mathbf{R}^{m}$. If $A$ is convex and compact, then $u_{A}$ and $w_{A}$ are continuous in the interior of $B=f_{*}(A)$, and in all of $B$ if $m=1$. Any right inverse $v$ must satisfy $u_{A} \leqslant v \leqslant w_{A}$ in $B$. In the complement of $B$ we have $u_{A}=+\infty, w_{A}=-\infty$.

We may ask whether there can exist a continuous right inverse even though the lower and upper right inverses are discontinuous. Maybe the simplest example to show this is the following, taken from [3, p. 63].

Example 4 Let $A$ be the convex hull of the two points $(1,0, \pm 1)$ and the circle

$$
\left\{(x, y, z) \in \mathbf{R}^{3} ; x^{2}+y^{2}=1, z=0\right\} .
$$

Then there is a continuous right inverse to the projection which forgets $z$, viz. $v(x, y)=(x, y, 0)$, and the upper and lower right inverses take the same values at $(x, y) \neq(1,0)$, but the values \pm 1 at $(1,0)$.

Example 5 It is also possible to construct an example with smooth boundary. Indeed, we may take the set in Example 3 with $b=0$. Then $u_{A} \leqslant 0 \leqslant w$, so $v_{A}(x, y)=0$ yields a continuous right inverse. But on the boundary of $B$, where 
$x^{2}+(y-r)^{2}=r^{2}$ (here $B$ is defined in (1)) we have $u_{A}(x, y)=w_{A}(x, y)=0$ when $x \neq 0$, whereas $u_{A}(0,1)=-1$ and $w_{A}(0,1)=1$.

Not only continuous functions but also semicontinuous functions, which we now proceed to define, are of importance here.

Definition 2 A function $f: \mathbf{R}^{m} \rightarrow \mathbf{R}_{\text {! }}$ is said to be lower semicontinuous if the strict finite superlevel set $\left\{x \in \mathbf{R}^{m} ; f(x) \in \mathbf{R}, f(x)>t\right\}$ is open in $\mathbf{R}^{m}$ for all real numbers $t$ (equivalently for all $t \in \mathbf{R}_{!}$).

To any function $f: \mathbf{R}^{m} \rightarrow \mathbf{R}_{\text {! }}$ we define its lower regularization $f_{b}$ (" $f$ flat"), defined as

$$
f_{\mathrm{b}}(x)=\liminf _{y \rightarrow x} f(y)=\sup _{r>0} \inf _{\|y\|<r} f(x+y), \quad x \in \mathbf{R}^{m} .
$$

By turning things upside down we define an upper semicontinuous function and its upper regularization $f^{\sharp}$ (" $f$ sharp"):

$$
f^{\sharp}(x)=\limsup _{y \rightarrow x} f(y)=\inf _{r>0} \sup _{\|y\|<r} f(x+y), \quad x \in \mathbf{R}^{m} .
$$

The mapping $f \mapsto f^{\sharp}$ is increasing for the usual order of functions ( $f \leqslant g$ implies $\left.f^{\sharp} \leqslant g^{\sharp}\right)$ and idempotent $\left(\left(f^{\sharp}\right)^{\#}=f^{\sharp}\right)$, so it is an ethmomorphism. Moreover it is larger than the identity $\left(f^{\sharp} \geqslant f\right)$ and so is a cleistomorphism.

\section{Squeezing in a continuous function between two given functions}

Proposition 1 Consider a function $f: \mathbf{R}^{m} \rightarrow \mathbf{R}_{!}$which is not identically equal to $+\infty$ and which satisfies an estimate

$$
f(x) \geqslant-C(1+\|x\|), \quad x \in \mathbf{R}^{m},
$$

for some constant $C>0$. Then we can approximate it from below by its infimal convolutions $f \sqcap(k V)$ with $k V$, where $V(x)=\|x\|$. More precisely, we define

$$
f_{k}(x)=(f \sqcap(k V))(x)=\inf _{y}(f(x-y) \uplus k V(y)), \quad x \in \mathbf{R}^{m}, \quad k>0 .
$$

Then $f_{k}$ has finite values when $k \geqslant C$ and is then Lipschitz continuous with Lipschitz constant $k$. The sequence increases to $f_{\mathrm{b}}$ as $k \nearrow+\infty$.

If we start with a function defined only on a subset of $\mathbf{R}^{m}$, we extend it by defining it to be $+\infty$ in the complement of its original domain of definition.

Proof The assumption that $f$ has at least one finite value, say at a point $c$, ensures that $f_{k}$ has values strictly less than $+\infty$ everywhere: $f_{k}(x) \leqslant f(c)+k V(x-c)<$ $+\infty$. 
The estimate (5) proves that $f_{k}$ does not take the value $-\infty$ if $k \geqslant C$, and also that it has Lipschitz constant $k$ since it is the infimum of a family of functions with Lipschitz constant $k$.

If $s<f(a)<+\infty$, then $f_{k}(a)>s$ for $k$ large enough, thus $f_{k}(a)$ comes arbitarily close to $f(a)$. If $f(a)=+\infty$ and $s<+\infty$, then $f_{k}(a)>s$, arbitrarily large for $k$ large.

Remark 1 In applications of this proposition it is sometimes convenient to replace $f$ by $\arctan f$, defined as $\pi / 2$ where $f=+\infty$ and as $-\pi / 2$ where $f=-\infty$, thus bounded and satisfying the hypotheses in the proposition. Of course the Lipschitz continuity of arctan $f$ has to be translated to continuity properties of $f$ with care.

Proposition 2 With $f$ as in Proposition 1, assume in addition that there is a compact set $K$ such that $f(x)=+\infty$ for all $x \notin K$. Then for any positive $\varepsilon, f_{k} \leqslant$ $f_{k+\varepsilon} \leqslant f_{k}+\varepsilon g$, where $g(x)=\sup _{y \in K}\|x-y\|, x \in \mathbf{R}^{m}$. In particular, $f_{k}$ depends continuously on $k \in \mathbf{R}, k>0$. The same is true if all points used in the construction of the approximation belong to a compact set.

The proof is an easy estimate. (If $f(x)=-\|x\|$, there is a discontinuity at $k=1$.)

Theorem 1 Let $u, v, w: B \rightarrow \mathbf{R}_{\text {! }}$ be three functions defined in a nonempty subset $B$ of $\mathbf{R}^{m}$. Assume that $u \leqslant v \leqslant w$ and that $v$ has finite values and is continuous. Then $\left.u^{\sharp}\right|_{B} \leqslant v \leqslant\left. w_{\mathrm{b}}\right|_{B}$.

Conversely, if $B \neq \varnothing$ is compact or open in $\mathbf{R}^{m}$ and if $u^{\#}<w_{\mathrm{b}}$ in $B$, now with strict inequality, then there exists a continuous function $v: B \rightarrow \mathbf{R}$ such that $\left.u^{\sharp}\right|_{B}<v<\left.w_{\text {b }}\right|_{B}$.

We shall consider the non-strict inequality $u^{\#} \leqslant w_{\mathrm{b}}$ later.

Proof Note that here the functions $u$ and $w$ are defined only in $B$, not in all of $\mathbf{R}^{m}$ as in (2). The functions $u^{\sharp}$ and $w_{\mathrm{b}}$ are therefore defined only in the closure of $B$, and to define them only values in $B$ are used. We state an inequality for the restrictions to $B$ of the functions but say nothing about their values on the possibly nonempty set $\bar{B} \backslash B$.

Since $u \leqslant v \leqslant w$ in $B$ we get $\left.u^{\sharp}\right|_{B} \leqslant\left. v^{\sharp}\right|_{B}=v$ and $v=\left.v_{b}\right|_{B} \leqslant\left. w_{b}\right|_{B}$.

For the second part we consider $w_{k}=w \sqcap(k V)$, which minorizes $\left.w_{\mathrm{b}}\right|_{B}$ and is continuous. For any given compact set $K \subset B$, we have $w_{k}>u^{\sharp}$ in $K$ for sufficiently large values of $k$. So if $B$ is compact we are done.

If $B$ is open, we can find a family $\left(K_{j}\right)_{j \in \mathbf{N}}$ of compact sets with union equal to $B$ and satisfying $K_{j} \subset K_{j+1}^{\circ}, j \in \mathbf{N}$. We define $K_{-1}$ to be the empty set. Then for smooth functions $\varphi_{j}$ satisfying $0 \leqslant \varphi_{j} \leqslant 1$, with support in the interior of $K_{j}$ and equal to 1 in $K_{j-1}$ we define $v=\varphi_{j} w_{k_{j}}+\left(1-\varphi_{j}\right) w_{k_{j+1}}$ in $K_{j} \backslash K_{j-1}$ (for each $x \in B$, there is a unique index $j \in \mathbf{N}$ such that $x$ belongs to $K_{j} \backslash K_{j-1}$ ). This yields a continuous function $v$ satisfying $u^{\#}<v<u_{\mathrm{b}}$ in all of $B$.

The methods in the last part of the proof can be used also for some sets $B$ which are neither compact nor open. 
We observe that the condition $u^{\sharp} \leqslant w_{b}$ is violated in Example 1: we have $u^{\sharp}(1,0)=2 \pi>0=w_{\text {b }}(1,0)$.

\section{Open sets}

If $A$ is open in $\mathbf{R}^{m}$, then $u_{A}$ as defined in Definition 1 is upper semicontinuous and $w_{A}$ is lower semicontinuous: $\left(u_{A}\right)^{\#}=u_{A}$ and $\left(w_{A}\right)_{b}=w_{A}$.

Theorem 2 Let $A$ be an open subset of $\mathbf{R}^{n}=\mathbf{R}^{m} \times \mathbf{R}$ (not necessarily convex), and let $f$ be the restriction to $A$ of the projection $\mathbf{R}^{n} \ni(x, t) \mapsto x \in \mathbf{R}^{m}$. Assume that the inverse image $f^{*}(\{x\})$ under $f$ of every point $x$ is connected. Then $f$ admits a continuous right inverse.

Proof The assumption on $A$ implies that the inverse image of a point $x$ in the image of $f$ is $\{x\} \times I_{x}$, where $I_{x}$ is a nonempty open interval; let us denote its endpoints by $u_{A}(x)$ and $w_{A}(x)$, so that $\left.I_{x}=\right] u_{A}(x), w_{A}(x)$ [. Moreover, as noted above, $u_{A}$ is upper semicontinuous and $w_{A}$ is lower semicontinuous.

If $u_{A}$ and $w_{A}$ have finite values, then the midpoint of $I_{x}$ defines a right inverse

$$
f_{*}(A) \ni x \mapsto\left(x, \frac{1}{2} u_{A}(x)+\frac{1}{2} w_{A}(x)\right) \in A,
$$

and it is continuous if $u_{A}$ and $w_{A}$ happen to be continuous. However, in general we have to define another kind of intermediate value between the two endpoints, a value which does not depend only on $u_{A}(x)$ and $w_{A}(x)$ but takes into account also points in a neighborhood of $x$. This is just what we did in Proposition 1 and Theorem 1.

See also Remark 1.

\section{Closed sets}

If $A$ is closed in $\mathbf{R}^{n}$, then $u_{A}$ is lower semicontinuous and $w_{A}$ is upper semicontinuous; we have $\left(u_{A}\right)_{\mathrm{b}}=u_{A}$ and $\left(w_{A}\right)^{\#}=w_{A}$.

We shall now adopt the methods to the case when the inverse image is a closed interval.

Theorem 3 Let $u, w: \mathbf{R}^{m} \rightarrow \mathbf{R}_{\text {! }}$ be two functions such that $-\infty<u^{\sharp} \leqslant w_{\mathrm{b}}<$ $+\infty$. Then there exists a continuous real-valued function $v$ such that $u^{\sharp} \leqslant v \leqslant w_{\mathrm{b}}$.

Proof If $\sup u \leqslant \inf w$, we can define a right inverse $v$ by taking its value as any constant $c$ satisfying $\sup u \leqslant c \leqslant \inf w$.

If instead we have $\sup u>\inf w$, we proceed as follows.

At a point such that $u^{\#}$ and $w_{\mathrm{b}}$ take the same value, we define $v(x)$ to be that value. 
Let $\Omega$ be the open set of all $x$ such that $u^{\sharp}(x)<w_{b}(x)$. We know that inf $w<$ sup $u$, so for small values of $k$ we have $w_{k}(x)<u^{\sharp}(x)$, while for large values of $k$, $w_{k}(x)>u^{\sharp}(x)$. We know that $w_{k}(x)$ increases to $w_{\mathrm{b}}(x)$ and even continuously so. We can apply Proposition 2: we note that the construction here is local, so that we can redefine $w$ to be $+\infty$ outside some compact set which contains all the points under consideration.

Therefore it is possible to choose a value for $k$ so that $w_{k}(x)$ is equal to for instance $w_{k}(x)=\frac{1}{2} u^{\sharp}(x)+\frac{1}{2} w_{b}(x)$.

Now the inequality $u^{\sharp}(y)<w_{k}(y)<w_{b}(y)$ holds not only for $y=x$ but for all $y$ in some neighborhood $\omega_{x}$ of $x$. The non-denumerable union of all the $\omega_{x}, x \in \Omega$, is equal to $\Omega$. A classical result on partitions of unity (see [5, p. 22], Théorème II or [1, p. 28], Theorem 1.4.5) now proves that there is a locally finite (thus denumerable) partition of unity $\left(\varphi_{j}\right)_{j \in \mathbf{N}}, \varphi_{j} \in \mathcal{D}\left(\omega_{j}\right)$, subordinate to the open covering $\left(\omega_{x}\right)_{x \in \Omega}$. We can define $v=\sum_{j \in \mathbf{N}} \varphi_{j} w_{k_{j}}$, where $k_{j}$ is the value chosen in $\omega_{j}$. This function $v$ is obviously a right inverse to the projection $\left(x_{1}, \ldots, x_{m}, x_{n}\right) \mapsto$ $\left(x_{1}, \ldots, x_{m}\right)$ and continuous by construction.

Theorem 4 Let $u, w: B \rightarrow \mathbf{R}$ be two bounded functions defined on a subset $B$ of $\mathbf{R}^{m}$. Then the following properties hold.

(a) There is a continuous function $v: B \rightarrow \mathbf{R}$ satisfying $u \leqslant v \leqslant w$ in $B$ if and only if $u^{\sharp} \leqslant w_{\mathrm{b}}$ in $B$.

(b) There is a continuous function $v: \bar{B} \rightarrow \mathbf{R}$ satisfying $u \leqslant v \leqslant w$ in $B$ if and only if $u^{\sharp} \leqslant w_{\mathrm{b}}$ in $\bar{B}$.

Proof Statement (a) in the direction if: If $u \leqslant v \leqslant w$ in $B$ and $v$ is continuous in $B$, we get $u^{\sharp} \leqslant v^{\sharp}=v_{\mathrm{b}} \leqslant w_{\mathrm{b}}$ in $B$, so that $u^{\sharp} \leqslant w_{\mathrm{b}}$ in $B$.

Statement (b) in the direction if: If $u \leqslant v \leqslant w$ in $B$ and $v$ is continuous in $\bar{B}$, we get $u^{\sharp} \leqslant v^{\sharp}=v_{\mathrm{b}} \leqslant w_{\mathrm{b}}$ in $\bar{B}$, so that $u^{\sharp} \leqslant w_{\mathrm{b}}$ in $\bar{B}$ (in the complement if $\bar{B}$ we have $u^{\sharp}=+\infty>-\infty=w_{b}$ ).

For the direction only if, we note that the result follows from Theorem 1 if $u$ and $w$ are bounded. If not, we consider $\arctan u$ and $\arctan w$ as explained in Remark 1. However, a priori it may then happen that $w_{k}$, which is constructed from $\arctan w$, takes the value $+\infty$, corresponding to $\pi / 2$ for $\arctan w_{k}$, for we have only non-strict inequalities $u^{\sharp} \leqslant w_{k} \leqslant w_{\mathrm{b}}$ in general. If so, the constructed function $v$ would not be real valued. To prove that we have strict inequality we note that, by hypothesis, $\arctan u^{\sharp}(x)<\arctan w_{b}(x)$ as soon as $\arctan w_{b}(x)=\pi / 2$ and $x$ is in the image of the projection. Then $w_{k}(x)<w(x)$ by Theorem 3 . The case when $u^{\sharp}(x)=-\pi / 2$ is similar. Hence we can be assured that $-\pi / 2<w_{k}(x)<\pi / 2$ for all $x$ in the image of $f$, so that $v$ is real valued and continuous.

We can now give a characterization of the sets and the projections that admit a continuous right inverse in the case of closed convex sets. 
Corollary 1 Let $A$ be a closed convex subset of $\mathbf{R}^{n}=\mathbf{R}^{m} \times \mathbf{R}$, let $f$ be the restriction to $A$ of the projection $\mathbf{R}^{n} \ni(x, t) \mapsto x \in \mathbf{R}^{m}$, and denote by $B$ the image of $A$ under $f$. Assume that there are two functions $u, w: B \rightarrow[-\infty,+\infty]$ such that

$$
A=\{(x, t) \in B \times \mathbf{R} ; u(x) \leqslant t \leqslant w(x)\} .
$$

Then $f$ admits a continuous right inverse if and only if $u^{\sharp} \leqslant w_{\mathrm{b}}$ on $B \backslash B^{\circ}$ and $u^{\sharp}<+\infty, w_{\mathrm{b}}>-\infty$ in $B$.

Proof Since $A$ is closed, $u$ is lower semicontinuous and $w$ is upper semicontinuous. However, due to the convexity, they are in fact continuous in the interior of $B$, so that $u^{\sharp}=u \leqslant w=w_{\mathrm{b}}$ there. The condition $u^{\sharp} \leqslant w_{\mathrm{b}}$ is therefore automatically satisfied in $B^{\circ}$, so we need require it only on a part of the boundary, viz. on $B \backslash B^{\circ}$.

That the conditions $u^{\#}<+\infty, w_{\mathrm{b}}>-\infty$ in $B$ are not superfluous is shown by the following example.

Example 6 Let $A$ be the set

$$
\left\{(x, y, z) \in \mathbf{R}^{3} ; y>0, z \geqslant x^{2} / y\right\} \cup\left\{(0,0, z) \in \mathbf{R}^{3} ; z \geqslant 0\right\} .
$$

This is a closed convex set. Indeed, it is the finite epigraph of the function

$$
f(x, y)=\sup _{\xi \in \mathbf{R}}\left(\xi x-\frac{1}{4} \xi^{2} y\right), \quad(x, y) \in \mathbf{R}^{2},
$$

i.e., the set $\left\{(x, y, t) \in \mathbf{R}^{2} \times \mathbf{R} ; t \geqslant f(x, y)\right\}$. The function $f$ is convex, being the supremum of a family of affine functions.

Define $u: \mathbf{R}^{2} \rightarrow[-\infty,+\infty]$ by $u(x, y)=x^{2} / y$ when $y>0, u(0,0)=0$, and $u(x, y)=+\infty$ otherwise. We have $\left.u\right|_{B}=\left.f\right|_{B}$ and $u_{\mathrm{b}}=f$ in $\mathbf{R}^{2}$. The set $B \backslash B^{\circ}$ consists of $\{(0,0)\}$ only, and $u^{\sharp}(0,0)=+\infty$, since $u(x, y) \geqslant x^{2} / y \geqslant x^{-2}$ if $y=x^{4}, x \neq 0$. So the condition $u^{\#}<+\infty$ in $B$ is violated, while the condition $u^{\sharp} \leqslant w_{\mathrm{b}}$ is satisfied, since $w_{\mathrm{b}}=+\infty$ in $B$. In $B^{\circ}$ there is a continuous right inverse to the projection which forgets $z$, but not in $B$, since any right inverse must satisfy $v(x, y) \geqslant u(x, y)$, and, as we just saw, $u(x, y)$ tends to $+\infty$ along a curve which approaches the origin.

If $A$ is bounded, the corollary can be simplified:

Corollary 2 Let $A$ be a compact convex subset of $\mathbf{R}^{m} \times \mathbf{R}$, let $f$ be the restriction to $A$ of the projection $\mathbf{R}^{m} \times \mathbf{R} \ni(x, t) \mapsto x \in \mathbf{R}^{m}$, and denote by $B$ the image of $A$ under $f$. Assume that there are two functions $u, w: B \rightarrow[-\infty,+\infty]$ such that

$$
A=\{(x, t) \in B \times \mathbf{R} ; u(x) \leqslant t \leqslant w(x)\} .
$$

Then $f$ admits a continuous right inverse if and only if $u^{\sharp} \leqslant w_{\mathrm{b}}$ on $\partial B$. 
Proof It is now clear that $u^{\sharp}$ and $w_{\mathrm{b}}$ are finite in $B$. Moreover, $B \backslash B^{\circ}=\partial B$, since $B$ is closed.

Theorem 5 Let $f: A \rightarrow B$ be as in Corollary 2. If the restriction of $f$ to the inverse image of $\partial B$ is injective (equivalently if $f^{*}(\{x\})$ is a singleton set for every $x \in \partial B$ ), then $f$ has a continuous right inverse; indeed, the lower and upper right inverses are continuous.

Thus injectiveness is sufficient, but by no means necessary, as shown by the polyhedra; see Theorem 6 below.

Proof That $\left.f\right|_{f^{*}(\partial B)}$ is injective means that the lower right inverse $u$ equals the upper right inverse $w$ on $\partial B$. The last coordinates satisfy

$$
u \leqslant u^{\sharp} \leqslant w^{\sharp}=w \text { and } u=u_{\mathrm{b}} \leqslant w_{\mathrm{b}} \leqslant w .
$$

At a point $a$ such that $u(a)=w(a)$ we must have $u^{\sharp}(a)=w_{b}(a)$, so the criterion in Corollary 2 is satisfied at all points $a \in \partial B$.

How arbitrary can the upper and lower right inverses be on the boundary $\partial B$ of $B$ ? The following result proves that they are quite arbitrary as long as $B$ is strictly convex, but must satisfy a very natural condition on flat pieces of the boundary of $B$.

Proposition 3 Let $B$ be a compact and convex subset of $\mathbf{R}^{m}$ and let two functions $u, w: \partial B \rightarrow \mathbf{R}$ be given, $u$ being lower semicontinuous and $w$ being upper semicontinuous with $u \leqslant w$. Assume that, for any supporting hyperplane $Y$, the restriction of $u$ to $Y \cap \partial B$ is convex, and that, similarly, the restriction of $w$ to $Y \cap \partial B$ is concave. Then there exists a compact convex set $A$ such that the lower right inverse of $f$ restricted to $\partial B$ is $x \mapsto(x, u(x))$ and the upper right inverse restricted to $\partial B$ is $x \mapsto(x, w(x))$.

Proof We define the lower surface of $A$ as the supremum of all affine functions $g$ : $\mathbf{R}^{m} \rightarrow \mathbf{R}$ such that $g(x) \leqslant u(x)$ for all $x \in \partial B$. The supremum $g$ of these functions is lower semicontinuous. The condition on $u$ guarantees that this supremum is equal to $u$ on $\partial B$. It is finite in $B$ and equal to $+\infty$ in the complement of $B$.

Similarly, the infimum of all affine functions $h: \mathbf{R}^{m} \rightarrow \mathbf{R}$ such that $h \geqslant w$ in $\partial B$ is equal to $w$ on $\partial B$.

\section{Polyhedra}

The origin of this section on polyhedra is a problem in digital geometry encountered by one of us; see Erik Melin's paper [2]. When approximating real-valued mappings by Khalimsky-continuous mappings, there are sometimes choices: we can introduce an upper and a lower approximation. However, for some real-valued mappings, the upper and the lower approximations are equal. 
In the paper mentioned it was proved that affine mappings have this property, and in the proof it had to be shown that two convex sets have non-empty intersection. This was done via the Brouwer fixed-point theorem. To construct the continuous functions involved, a slightly weaker version of the following result was needed.

Proposition 4 Let $F$ be a linear mapping of $\mathbf{R}^{n}$ into $\mathbf{R}^{m}$ and let $A$ be a compact convex subset of $\mathbf{R}^{n}, B$ its image under $F$. Assume that the restriction of $F$ to $A$ possesses a continuous right inverse. Then the same is true for any set $A_{1}$ which is the convex hull of $A$ and finitely many points.

Proof By induction it is enough to let $A_{1}$ be the convex hull of the union of $A$ and a single point $a$ such that $F(a)$ does not belong to $B$, thus $A_{1}=\operatorname{cvxh}(A \cup\{a\})$, the convex hull of $A \cup\{a\}$. Given a right inverse $v: B \rightarrow A$, one then defines a right inverse $v_{1}: B_{1} \rightarrow A_{1}$ to $f_{1}=\left.F\right|_{A_{1}}$, where $B_{1}=\operatorname{cvxh}(B \cup\{F(a)\})$, by taking $v_{1}=v$ in $B, v_{1}(F(a))=a$, and $v_{1}$ as an affine function on each segment between $F(a)$ and a point in $\partial B$, more precisely the first point in $\partial B$ when going from $a$ through the given point. In fact, any point $x \in B_{1} \backslash B$ can be written uniquely as $x=(1-t) F(a)+t y$ for some $t$ satisfying $0 \leqslant t<1$, where $y$ belongs to $B$ and is such that $(1-s) F(a)+s y \notin B$ when $0 \leqslant s<1$. (Then necessarily $y$ belongs to $\partial B$.) We then set $v_{1}(x)=(1-t) a+t v(y)$.

Theorem 6 With $f: A \rightarrow B$ as in Proposition 4, assume in addition that $A$ is a polyhedron. Then the upper and lower right inverses are Lipschitz continuous.

Proof That $A$ is a polyhedron means that it can be described as the intersection of a finite family of closed half spaces, say

$$
\left\{x \in \mathbf{R}^{n} ; \xi^{(j)} \cdot x \leqslant c_{j}\right\}, \quad j \in J,
$$

where $J$ is a finite index set. We may assume that $\left(\xi^{(j)}\right)_{n}$ is either $1,-1$ or 0 . Let $J_{[-1]}$ be the set of those $j$ for which $\left(\xi^{(j)}\right)_{n}=-1$. Then the last coordinate of the lower right inverse $u=\left(u_{1}, \ldots, u_{n}\right)$ is

$$
u_{n}\left(x_{1}, \ldots, x_{n-1}\right)=\sup _{j \in J_{[-1]}}\left(\xi_{1}^{(j)} x_{1}+\cdots+\xi_{n-1}^{(j)} x_{n-1}-c_{j}\right), \quad x \in B,
$$

thus a supremum of a finite family of affine functions, hence Lipschitz continuous.

\section{A sufficient condition for injectivity of a projection}

The support function of a subset $A$ of a vector space $E$ is

$$
H_{A}(\xi)=\sup _{x \in A} \xi \cdot x, \quad \xi \in E^{*},
$$

where $E^{*}$ is the algebraic dual of $E$. When $E=\mathbf{R}^{m}$ we identify $E^{*}$ with $\mathbf{R}^{m}$. 
Proposition 5 Let $A$ be a compact convex subset of $\mathbf{R}^{n}$, let

$$
f: A \rightarrow B, f\left(x_{1}, \ldots, x_{n}\right)=\left(x_{1}, \ldots, x_{n-1}\right),
$$

be as before, and assume that there exists a compact convex subset $A_{0}$ of $A$ such that $f_{*}\left(A_{0}\right)=f_{*}(A)=B$ and such that its support function $H_{A_{0}}$ satisfies

$$
\frac{H_{A_{0}}\left(\xi^{\prime}, t\right)-2 H_{A_{0}}\left(\xi^{\prime}, 0\right)+H_{A_{0}}\left(\xi^{\prime},-t\right)}{2 t} \nrightarrow 0 \text { as } t \neq 0, t \rightarrow 0 .
$$

Then $f$ has a continuous right inverse $v: B \rightarrow A$ such that $\operatorname{graph}(v) \subset A_{0}$.

Proof The limit of the quotient $\left(H_{A_{0}}\left(\xi^{\prime}, t\right)-H_{A_{0}}\left(\xi^{\prime}, 0\right)\right) / t$ as $t$ tends to zero along positive values is equal to the derivative to the right at $\left(\xi^{\prime}, 0\right)$. The difference between this limit and the derivative from the left is the kink in the graph, and condition (7) ensures that the hypothesis on injectivity in Theorem 5 is satisfied.

\section{The exceptional character of bad projections}

We have seen projections that do not admit a continuous right inverse. Are they rare in any sense? We shall prove a result in this direction here.

Theorem 7 Let $A$ be a convex compact set in $\mathbf{R}^{n}, n \geqslant 3$, and fix an affine subspace $E$ of dimension 3 in $\mathbf{R}^{n}$. Assume that $A \cap E$ has a nonempty interior in $E$, and let $\alpha$ be any unit vector in $E$. Let $\beta$ be any unit vector in $E$ orthogonal to $\alpha$ and define $f_{\beta}$ to be the projection from $A \cap E$ along $\beta$ to the two-dimensional plane $Y_{\beta} \subset E$ of equation $\beta \cdot x=0$.

There are only denumerably many $\beta$ such that $f_{\beta}$ does not have a continuous right inverse.

Proof We may assume that the origin belongs to the interior of $A$ relative to $E$. Let $p$ be any point on the boundary of $A \cap E$ relative to $E$ and assume that there is no continuous right inverse at the point $f_{\beta}(p) \in Y_{\beta}$ for the projection $f_{\beta}$ along $\beta$. Then we know from Theorem 3 that there is a segment of positive length in the boundary of $A \cap E$ relative to $E$ which is mapped to $f_{\beta}(p)$. Now the boundary of the two-dimensional convex set $A \cap Y_{\beta}$ has finite length, and so can only contain denumerably many such segments, whereas there are $\operatorname{card}(\mathbf{R})>\operatorname{card}(\mathbf{N})$ different projections defined by $\beta$ in total.

Acknowledgements We are sincerely grateful to Göran Björck, Jan Boman and Gerd Brandell for corrections, constructive criticism and helpful suggestions to earlier versions of this paper. We thank Hiroshi Yamaguchi for encouragement over a number of years.

An anonymous referee has sent us most valuable corrections and recommendations, for which we are sincerely grateful. The report enabled us to improve the presentation.

Funding Open access funding provided by Uppsala University. 
Open Access This article is licensed under a Creative Commons Attribution 4.0 International License, which permits use, sharing, adaptation, distribution and reproduction in any medium or format, as long as you give appropriate credit to the original author(s) and the source, provide a link to the Creative Commons licence, and indicate if changes were made. The images or other third party material in this article are included in the article's Creative Commons licence, unless indicated otherwise in a credit line to the material. If material is not included in the article's Creative Commons licence and your intended use is not permitted by statutory regulation or exceeds the permitted use, you will need to obtain permission directly from the copyright holder. To view a copy of this licence, visit http://creativecommons.org/licenses/by/4. $0 /$.

\section{References}

1. Hörmander, L.: The Analysis of Linear Partial Differential Operators, 1. Distribution Theory and Fourier Analysis. 2nd edn. Springer, Berlin (1990)

2. Melin, Erik. 2005. Extension of continuous functions in digital spaces with the Khalimsky topology. Topology Appl. 153, no. 1, 52-65

3. Наймарк, М.А.: Нормированные кольца. Государственное издательство техническотеорической литературы, Москва (1956)

4. Repovš, D., Semenov, P.V.: Continuous selections of multivalued mappings, viii +356 pp. Mathematics and its Applications, 455. Kluwer Academic Publishers, Dortrecht (1998)

5. Schwartz, L.: Théorie des distributions. Hermann, Paris (1966) 